\title{
SERJANIA SETIGera (SAPINDACEAE), NUEVo REgistro PARA LA Flora DE BOLIVIA
}

\author{
JUAN PABLO COULLERI ${ }^{1,2}$ y MARÍA SILVIA FERRUCCI ${ }^{1}$
}

\begin{abstract}
Summary: Serjania setigera (Sapindaceae, Paullinieae), new record for the flora of Bolivia. Serjania setigera Radlk. (Sapindaceae), species known for Mato Grosso do Sul State, is mentioned for the first time for the Bolivian flora; it was collected on the border between Bolivia and Brazil. In this paper a complete description, a first iconography and a distribution map of this species is provided. This new record increases knowledge of the biodiversity of ecosystems that occur on calcareous soils present in the wetlands surrounding the Brazilian Pantanal.
\end{abstract}

Key words: Bolivia, calcareous soils, liana, new record, Sapindaceae, Paullinieae.

Resumen: Serjania setigera Radlk. (Sapindaceae, Paullinieae), especie conocida para el estado de Mato Grosso do Sul, se cita por primera vez para la flora boliviana; la misma fue coleccionada en el límite entre Bolivia y Brasil. Se proporciona una descripción completa, una primera iconografía y un mapa de distribución. Este nuevo registro incrementa el conocimiento de la biodiversidad de los ecosistemas de suelos calcáreos presentes en las tierras húmedas que rodean al pantanal brasileño.

Palabras clave: Bolivia, liana, suelos calcáreos, nuevo registro, Sapindaceae, Paullinieae.

\section{INTRODUCCIÓN}

Serjania Mill. es el género más importante de la tribu Paullinieae en cuanto a número de especies, con aproximadamente 231 especies (Ferrucci $\&$ Coulleri, 2013). Es un género americano con distribución tropical a subtropical, está representado desde el sur de Estados Unidos hasta el centro de Argentina. Los aportes más significativos a la taxonomía del género fueron realizados por Radlkofer (1874, 1875), quien en 1874 publica la sinopsis del género, en la cual segrega las especies en 12 secciones basándose principalmente en caracteres carpológicos y florales; en 1875 publica la monografía del mismo. Recientemente Acevedo-Rodríguez (1993) redujo las 12 secciones de Radlkofer a 6 (Tabla 1), reconociendo solo 5 secciones originales y fundando una nueva sección,

\footnotetext{
1 Instituto de Botánica del Nordeste (UNNE-CONICET), C.C. 209, 3400-Corrientes, Argentina.

2 E-mail: juancoulleri@gmail.com
}

esta clasificación tiene en cuenta únicamente caracteres carpológicos y seminales.

Brasil con 119 spp. (Somner et al., 2015), es el centro de mayor diversidad del género seguido de Bolivia que de acuerdo al tratamiento de la familia para el Catálogo de Plantas Vasculares de Bolivia reconoce 57 especies, 2 de estas endémicas (Ferrucci et al., 2014). De acuerdo al criterio de Radlkofer (1931-1932) estas 57 especies están segregadas en 11 secciones, la única que aparentemente no estaba representada era la sección Ceratococcus Radlk. Con respecto a las secciones propuestas por Acevedo-Rodríguez, en Bolivia estarían presentes especies de 5 secciones, con la misma observación sobre la sección Ceratococcus. La posibilidad de coleccionar recientemente material de $S$. setigera Radlk. en el límite entre Bolivia y Brasil, permitió tener representadas en Bolivia el total de la secciones del género.

El objetivo de este trabajo es dar a conocer el primer registro de $S$. setigera para Bolivia; se la describe y se indica su distribución geográfica, además se la ilustra por primera vez. 


\section{Materiales y Métodos}

Los datos presentados se obtuvieron del estudio de una colección realizada en Puerto Quijarro (Dpto. Santa Cruz, Provincia G. Busch, Bolivia) en julio de 2013 que fuera depositada en el herbario CTES, de un obsequio enviado por el herbario CPAP y de los ejemplares depositados en el herbario CTES. El estudio morfológico se llevó a cabo con microscopio estereoscópico (Leica MZ 6), y se consultó la descripción original y el ejemplar tipo. El mapa de distribución se realizó sobre la base del material revisado.

\section{Resultados}

Serjania setigera Radlk., Repert. Spec. Nov. Regni Veg. 17: 356. 1921. "In Brasiliae civitate Mato Grosso: Malme, $n^{\circ} 3052$ ! (Corumba, m. Apr. 1903; Hb. Holm.)". Tipo: Brasil. Mato Grosso do Sul. Corumbá, 6-IV-1903, fl. \& fr., Malme G. O. A. 03052 (holotipo S! 3 cartulinas). Fig. 1.

Frútice trepador setoso. Indumento en dos estratos, setas ferrugíneas de hasta 3-3,5 mm en las costillas y pelos simples breves en los surcos en el tallo, setas de menor longitud y pelos simples entremezclados en ejes de la hoja e inflorescencia. Tallo castaño, anguloso 6-costado, con 3 costillas prominentes determinadas por 3 cámbiumes supernumerarios equidistantes. Hojas: estípulas escariosas, reflexas, ovado-obtusas, 0,4-0,6 x 0,5$0,9 \mathrm{~cm}$, persistentes, setulosas en la cara abaxial, glabras en adaxial; pecíolo subterete, ventralmente canaliculado, 3,5-5,5 cm long., setuloso; raquis bicanaliculado, estriado, 2,5-4 cm long.; lámina 2-yugada, 5-foliolada; folíolos cartáceos, venación craspedódroma simple, las terciarias con patrón percurrente; peciólulo del folíolo terminal de 0,9-2 cm, en los demás menor a subsésiles; folíolo terminal ovado-romboidal, 4-9 x 2,7-6 $\mathrm{cm}$, base decurrente, margen inciso-dentado, ciliado, dientes agudos u obtusos glandulares, ápice acuminado raro agudo; folíolos laterales ovados, 4-8 x 1,3-5 cm, base redondeada o cuneada, margen similar al folíolo terminal, ápice agudo raro obtuso; epifilo pubérulo, pelos subsetáceos adpresos, hipofilo subvelloso. Inflorescencia: tirso simple, axilar, espiciforme o subespiciforme; pedúnculo subcuadrangular, pubescente, 4,1-10 cm long.; raquis subterete, pubescente, $3,4-8 \mathrm{~cm}$ long.; cincinos sésiles o subsésiles con pedicelos muy cortos de hasta $1 \mathrm{~mm}$ long.; pedicelo floral 2,5-4 mm long., en el fruto 3-5 mm long., ambos articulados en la base; brácteas ovado-oblongas, 0,5-0,7 cm long., pubescentes solo en la cara abaxial, bractéolas conformes, menores. Flores: ca. $10 \mathrm{~mm}$ long., con sépalos externos ovado-oblongos, $4,5 \times 2,5 \mathrm{~mm}$, pubescentes en la cara abaxial, pubérulos en adaxial y con pelos glandulares en los márgenes, los internos ovado-angostos, 5-7 x 2,5 $\mathrm{mm}$, pubescentes en ambas caras; pétalos obovadounguiculados, glandulosos en la cara adaxial, los posteriores 7-9,5 x 4,5-5,5 mm, apéndice ca. 5,5 x $3,6 \mathrm{~mm}$, con la cresta bipartida, los anteriores 7-9,5 x 4,5-4,7 mm, apéndice ca. 5,5 x 3,2 mm; lobos nectaríferos posteriores ovoide-obtusos o transverso-oblongos, pubérulos, los anteriores redondeados, menores, glabros; androginóforo glabro; flor estaminada con estambres de hasta 3 $\mathrm{mm}$ long., filamentos pilosos, pistilodio ca. 1,5 mm long.; en la flor pistilada, estambres con filamentos pilosos, ca. 2,5 mm long., estilo bastante más largo que ramas del estigma (caracteres observados en fruto joven). Fruto: ovado-cordado, cartáceo, sin constricción debajo de la porción seminífera, mericarpo de 3-4 x 1,8-2,25 cm, a la altura del lóculo 1,2-1,5 cm lat., con cresta dorsal ca. $0,3 \mathrm{~cm}$ lat., con una proyección lateral del septo, a la altura del lóculo de 0,3-0,5 cm lat., lóculo hinchado; epicarpo con porción seminífera setulosa, venación marcada, castaño-oscura, el ala castaño-claro, pubescente con indumento de pelos simples, endocarpo glabro. Semilla trígono-obovoidea, inserta debajo de la mitad del lóculo. $2 \mathrm{n}=24$ (Ferrucci \& Solís Neffa, 1997).

Distribución y hábitat: Estaba registrada como endémica del pantanal, MS, Brasil (Somner et al., 2010). El ejemplar coleccionado en Bolivia, en el sureste del departamento de Santa Cruz, en el límite con Mato Groso do Sul, al sur de Puerto Suarez permite citarla por primera vez para el país (Fig. 2). La distribución en Bolivia ubica a esta especie en la región fitogeográfica del Bosque Semideciduo Chiquitano.

Fenología: Florece de diciembre a abril y con frutos maduros de mayo a julio. 


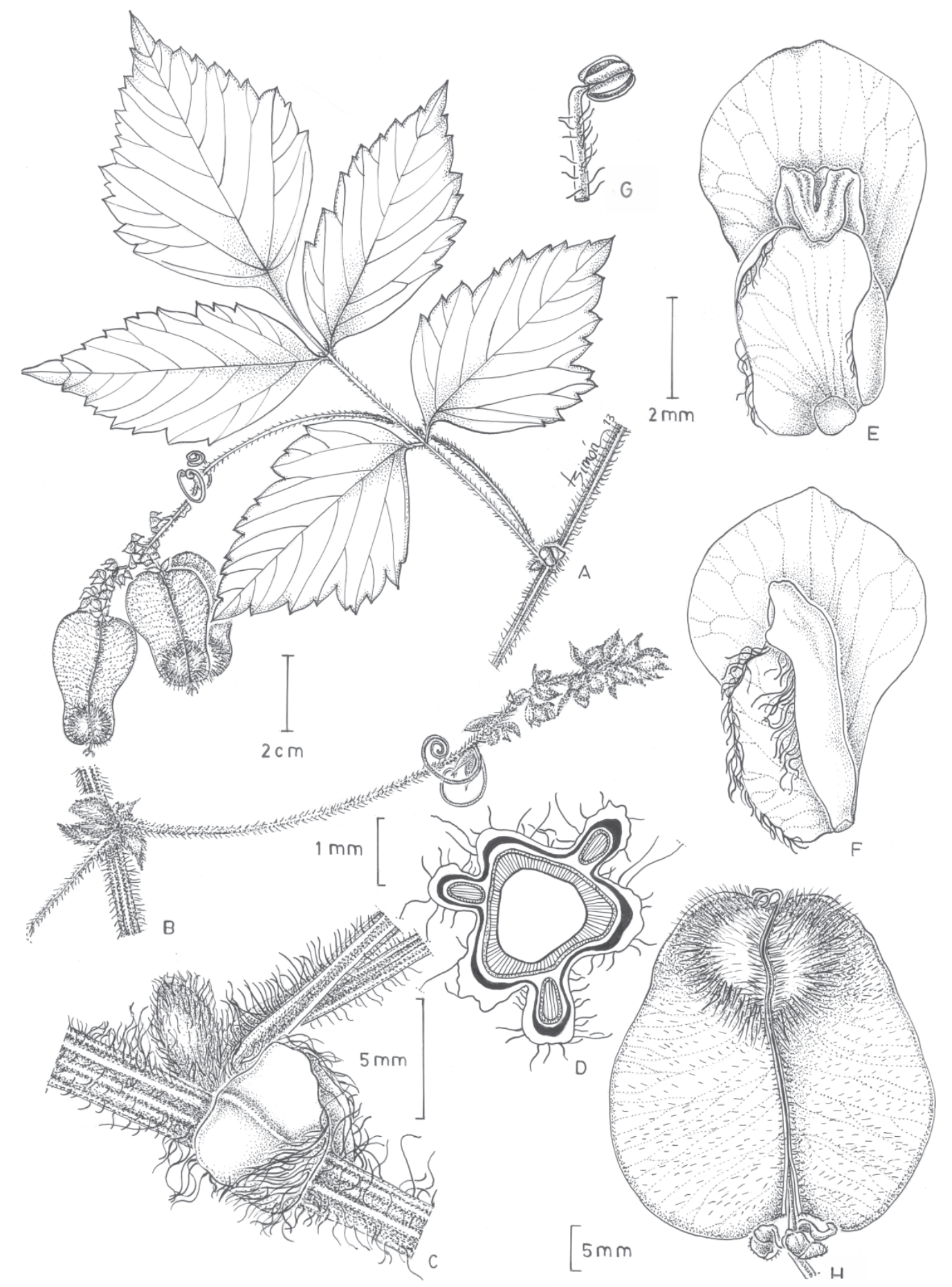

Fig. 1. Serjania setigera. A: Rama fructífera. B: Detalle de la inflorescencia. C: Detalle de las estípulas. D: Corte transversal del tallo. E: Pétalo posterior, cara adaxial. F: Pétalo anterior, cara adaxial. G: estambre de la flor estaminada. H: fruto casi maduro. (A, Coulleri et al. 568 (CTES); B, D, Jesus et al. 487 (CTES); C, H. Pott A. et al. 7818 (CTES); E-G. Jesus et al. 411 (CTES). 


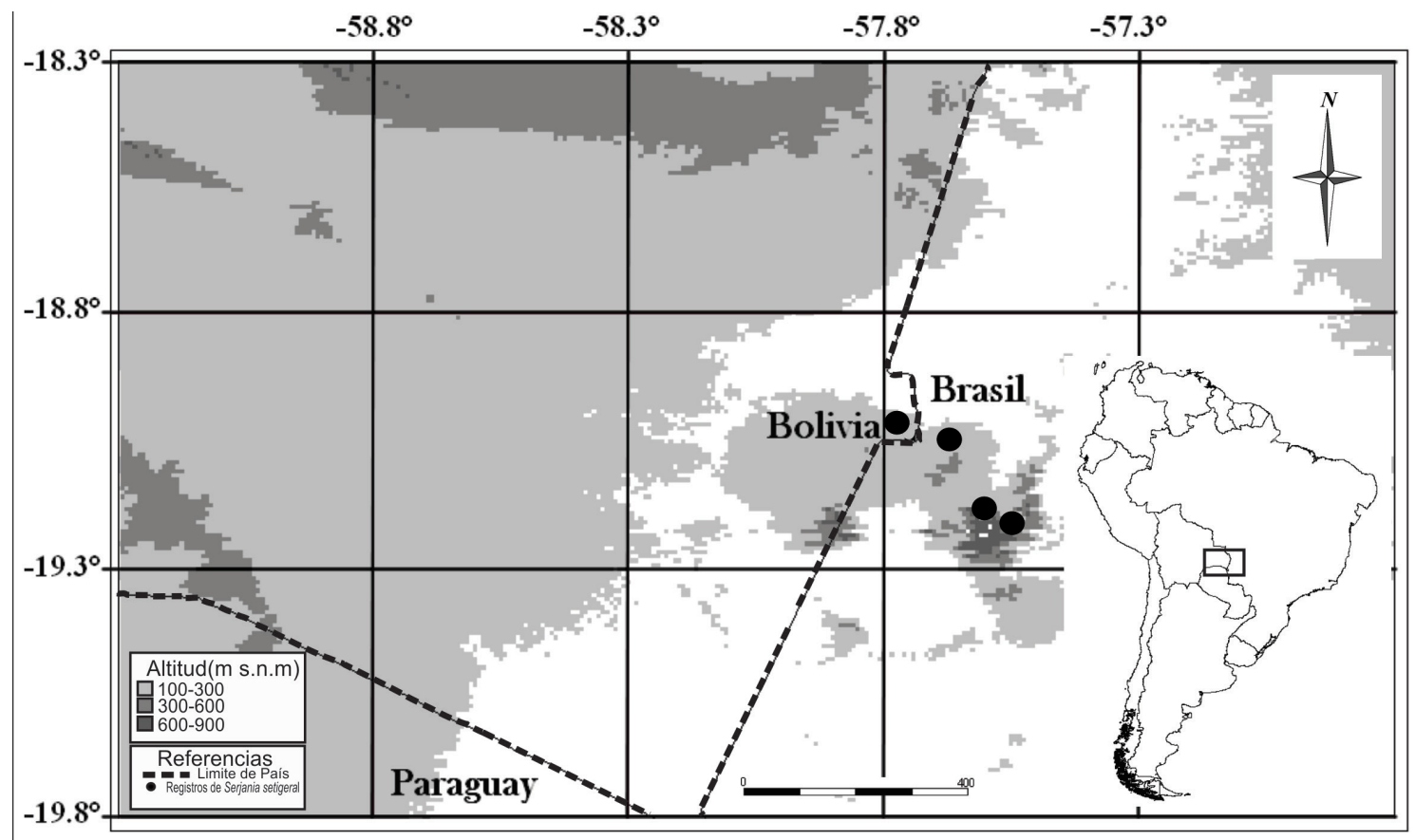

Fig. 2. Distribución geográfica de S. setigera, especie endémica de suelos calcáreos.

Material adicional examinado: BOLIVIA. Santa Cruz: Provincia G. Busch, Puerto Quijarro, $0,7 \mathrm{~km} \mathrm{~S}$ del puesto fronterizo, $19^{\circ} 02^{\prime} 2,2^{\prime \prime} \mathrm{S}$, $57^{\circ} 42^{\prime} 37^{\prime \prime} \mathrm{W}$, selva en galería, 20-VII-2013, fr., Coulleri, J.P. et al. 568 (CTES). BRASIL. Mato Grosso do Sul: Mun. Corumbá, Assentamento Tamarineiro II Sul, Sitio Sr. Albino lote 92, trihla 3, beira de mata decídua, solo argiloso, $19^{\circ} 06^{\prime} 31^{\prime \prime} \mathrm{S}$, 57047'15' W, 153 m m.s., 07-I-2009, fl., Jesus, E.M. et al. 411 (CPAP, CTES); idem, 18-III-2009, fr inmaduro, Jesus, E.M. et al. 487 (CPAP, CTES); Estrada do Tamarindeiro, beira de corrego, solo calcimórfico, $110 \mathrm{~m}$ m.s., 19 ${ }^{\circ}, 06^{\prime} \mathrm{S}, 57^{\circ} 47^{\prime} \mathrm{W}, 23-$ V-1987 (fr.), Pott, A. et al. 2730 (CPAP, CTES); Entre fazenda Alvorada e fazenda Noroeste, mata com Aspidosperma (quebracho), Calycophyllum, solo argiloso calcimórfico, transição entre pantanal e morraria calcária, 150 m m.s., 24-V-1989, fr., Pott, A. 4875 (CPAP, CTES); $1 \mathrm{Km}$ sul do posto Esdras na estrada do Tamarineiro, junto a divisa Brasil-Bolivia, $19^{\circ} 02^{\prime} 03^{\prime \prime} \mathrm{S}, 57^{\circ} 42^{\prime} 33^{\prime \prime} \mathrm{W}, 100 \mathrm{~m}$ m.s., solo argiloso calcimórfico, 12-VI-1996 (fr.), Pott, A. et al. 7818 (CPAP, CTES); Fazenda Água Verde, litossolo, $19^{\circ} 01^{\prime} \mathrm{S}, 57^{\circ} 39^{\prime} \mathrm{W}, 08-\mathrm{II}-1990$, fl., Pott, V.J. et al. 1271 (CPAP, CTES); Mun. Ladário, fazenda Vale do Paraíso, morro Sta. Cruz, $19^{\circ} 10^{\prime} 26,8^{\prime \prime} \mathrm{S}, 57^{\circ} 34^{\prime} 44,9^{\prime \prime} \mathrm{W}, 200 \mathrm{~m}$ m.s., mata decídua, solo litólico, 15-III-2001, fr. inmaduro, Damasceno Junior, G.A. \& Assis, E.M. 2242 (COR, CTES); Mun. Ládario, fazenda São João, $19^{\circ} 11^{\prime} 21,2 \mathrm{~S}, 57^{\circ} 31^{\prime} 43,9^{\prime \prime} \mathrm{W}, 200 \mathrm{~m}$ m.s., mata decídua, solo litólico, 16-VI-2001, fr., Damasceno Junior, G.A \& Velasques, J.S. 2451 (COR, CTES).

Esta especie pertenece a la sección Ceratococcus, es el primer registro de esta sección en Bolivia. La misma se caracteriza por presentar una proyección lateral corniculiforme a la altura de la porción seminífera, a excepción de la especie aquí descripta. Serjania setigera no presenta esta característica, sin embargo, exhibe una proyección lateral del septo notable, no observada en el resto de las especies presentes en Bolivia.

El hallazgo de $S$. setigera en territorio boliviano además de constituir un nuevo aporte al conocimiento de la flora de ese país, permite tener representadas en Bolivia todas las secciones del género, independientemente del criterio de clasificación infragenérica escogido (Radlkofer, 


\section{J. P. Coulleri y M. S. Ferrucci - Serjania setigera en Bolivia}

1931-1932; Acevedo-Rodríguez, 1993). Además, también se evidencia que esta especie sería una especie endémica de suelos calcáreos como los que ocurren en las regiones del este del departamento Santa Cruz (Bolivia) y en las inmediaciones de Corumbá (Mato Grosso do Sul, Brasil) (Salis et al. 2004). Esta área de suelos calcáreos tanto de Bolivia como de Brasil, además son zonas que no se inundan y sirven de refugio para la fauna durante la época de inundaciones del pantanal (Carvalho et al., 2002), por lo cual son de suma importancia en cuanto a la conservación de estos ecosistemas, situación que no se observa debido a la extensión del cultivo de soja de la última década (Fearnside, 2001), y a la utilización de la vegetación correspondiente a los campos cerrados, contiguos a estos sistemas de suelos calcáreos, como terrenos de pasturas (Salis et al., 2004).

De esta manera, el conocimiento de la biodiversidad de estos ecosistemas permitirá en el futuro establecer las prioridades de conservación y tomar las acciones necesarias para evitar la degradación de éstos.

\section{Agradecimientos}

Los autores agradecen a la Myndel Botanica Foundation por financiar los viajes de colección realizados a Bolivia que permitieron el desarrollo no solo de este, sino de varios trabajos de investigación; al Consejo Nacional de Investigaciones Científicas y Técnicas; al Herbario do EMBRAPA Pantanal (CPAP),y a los Dres. Massimiliano Dematteis y Juan Domingo Urdampilleta quienes participaron del viaje de colección.

\section{Bibliografía}

ACEVEDO-RODRÍGUEZ, P. 1993. Systematics of Serjania (Sapindaceae). Part I: A revision of Serjania Sect. Platycoccus. Mem. New York Bot. Gard. 67: $1-93$.

CARVALHO, M. P., R. P. SORATTO \& O. S. FREDDI . 2002. Variabilidade espacial de atributos físicos em um latossolo vermelho distrófico sob preparo convencional em Selvíria, Estado de Mato Grosso do Sul. Acta Sci. 24: 1353-1361.
FEARNSIDE, P. M. 2001. Soybean cultivation as a threat to the environment in Brazil. Environ. Conserv. 28: 23-38.

FERRUCCI, M. S. \& V. G. SOLÍS NEFFA. 1997. Citotaxonomía de Sapindaceae Sudamericanas. Bol. Soc. Argent. Bot. 33:77-83

FERRUCCI, M. S. \& J. P. COULLERI. 2013. Serjania lucianoi (Sapindaceae: Paullinieae), a new species from Northern Bahia, Brazil. Syst. Bot. 38: 172-177.

FERRUCCI, M. S. \& P. ACEVEDO-RODRÍGUEZ. 2014. Sapindaceae pp.1181-1192. In: JØRGENSEN, P.M., M. H. NEE \& S.G. BECK. Catálogo de las plantas vasculares de Bolivia. St. Louis: Missouri Botanical Garden Press,

RADLKOFER, L. 1874. Conspectus sectionum specierumque generis Serjaniae. F. Straub, München.

RADLKOFER, L. 1875. Monographie der SapindaceenGattung Serjania. Verlag der Königl. Bayer. Akademie, München.

RADLKOFER, L. 1931. Sapindaceae. In: ENGLER, A. (ed.), Das Pflanzenreich IV, 165 (Heft 98a-h), pp. 1-219. Verlag von Wilhelm Engelmann, Leipzig.

SALIS, S. M., M. PEREIRA DA SILVA, P.P. DE MATTOS, J. S. VILA DA SILVA, V. J. POTT \& A. POTT. 2004. Fitossociologia de remanescentes de floresta estacional decidual em Corumbá, Estado do Mato Grosso do Sul, Brasil. Revista. Brasi. Bot. 27: 671-684.

SOMNER, G. V., M. S. FERRUCCI, P. ACEVEDORODRÍGUEZ \& G. R. COELHO. 2010. Sapindaceae. pp. 1606-1620. In: CAMPOSTRINI FORZZA, R. \& P. LEITMAN (coord.), Catálogo de Plantas e Fungos do Brasil. Vol. 2. Angiospermas. Pp. 868-1699. Gráfica Santa Marta. Jardim Botânico do Rio de Janeiro.

SOMNER, G.V., M.S. FERRUCCI \& P. ACEVEDORODRÍGUEZ. Serjania. In: Lista de Espécies da Flora do Brasil. Jardim Botânico do Rio de Janeiro. Disponible en: <http://floradobrasil.jbrj.gov.br/ jabot/floradobrasil/FB20937>.

Recibido el 2 de junio de 2015, aceptado el 11 de septiembre de 2015 . 
